\title{
Digital Ortho-image Production for Web GIS Applications
}

\author{
Hong-Gyoo Sohn, Hyo-Keun Park, Choung-Hwan Park, and Joon Heo \\ School of Civil and Env. Eng., Yonsei University, Seoul, Korea \\ \{sohn1, bear0514, c142520, heo\} ayonsei.ac.kr
}

\begin{abstract}
This paper presents a true ortho-image generation scheme in urban areas for web GIS application such as urban management system. Proposed scheme includes the solution of conventional problems in normal ortho-image generation. Especially, our approach is focused on the automation and consistency of ortho-image in complex urban areas. For this purpose, we generated additionally plotted DSM (Digital Surface Model), which includes roads, highlevel roads, bridges, and tunnels, automatically detected occlusion pixels and restored occlusion areas using a self-developed algorithm, and performed mosaic process using road-based seamlines. Total 2,677 aerial color images were used to create the so called true ortho-image map for Seoul metropolitan city. It turned out that the proposed method could generate true ortho-image map for urban areas with high positional accuracy and good visual consistency. The generated ortho-image database is being successfully operated by Seoul Metropolitan Aerial Photography Management Systems on the web and urban management systems.
\end{abstract}

\section{Introduction}

With recent technical advance in photogrammetry and GIS (Geospatial Information System), the digital ortho-image has become an integral part of geo-spatial data. This is because ortho-images not only have the coordinate system like a traditional map but also provide the visual information of ground object. Accordingly digital ortho-image is commonly used as a framework data in GIS [1]. For example, high-resolution satellite image and color aerial image have been extensively used to construct urban information systems [2]. In general, the ortho-image for GIS applications needs several complex photogrammetric processes. These include three essential processes: 1) Differential rectification; 2) Mosaicking; and 3) Image compression.

Differential rectification involves relating the digital image to actual terrain coordinates and elevations (e.g., a Digital Elevation Model (DEM)) so as to correct for scale variation due to terrain relief and image displacement due to the angular offsets of aerial camera at the time of exposure [3]. Differential rectification is the essential process in ortho-image generation. It requires special algorithms to keep positional accuracy. Also, it often needs accurate DEM as well as Digital Surface Model (DSM) for target areas. Here we differentiate DEM and DSM. DEM means the elevation of terrain itself but DSM means terrain elevation including surface features such as buildings, bridge, and trees. 
Second, for large scale ortho-image compilation, it is often efficient and convenient to perform differential rectification on each image independently, and to put them together in the later step. This process is known as mosaicking. Detecting and placing seamlines in consecutive images is the important technique in mosaicking process [4]. Finally, for dealing with large volumes of image data sets most GIS applications require the image compression process on the generated ortho-images. The image compression is another critical step for the GIS systems operated via internet.

For the past two decades most of studies have mainly focused on the processes of ortho-image generation [5]. In this paper, we presented an effective "true orthoimage" generation scheme for the urban management system on the web. Here "true" refers to differential rectification of input image including man-made surface features such as buildings and brides. In particular, we provided the solution for critical problems that occur in ortho-image generation for urban areas, which improves positional accuracy and visual consistency of true ortho-image. To test our scheme, 2,677 aerial images were used to construct ortho-image database for Seoul metropolitan city. The ortho-image database generated from this project is being used in "Seoul Aerial Photography Management Systems" on the web, whose purpose is to manage Seoul metropolitan city more efficiently (e.g., facility management and illegal building detection, etc).

\section{True Ortho-image Solution}

Aerial imagery acquired by a perspective projection shows a situation projected through perspective center onto the image plane. As a result, some objects on the image placed at the same point but at different elevation will be projected to different positions in the aerial imagery. This phenomenon is called relief displacement. As an effect of these relief displacements, objects which are displaced at a high elevation also look relatively bigger in the image. Besides various geometric distortions by flight attitude exist on image. Therefore, a special process for generating image which has ortho-geometry such as a map is needed. It can be accomplished by digital differential rectification [6]. Ortho-rectification process means a process that tries to eliminate the perspective characteristics of image. The result image has an ortho projection where all rays are parallel and shows a situation where the perspective aspect of image has been removed. Therefore, ortho-rectified image has a consistent scale and can be used as a planimetric map. This makes it useable for combining with geo-spatial data in GIS or as part of 3-D visualization.

\subsection{Inherent Problems in Digital Ortho-image Production of Urban Areas}

A conventional problem for ortho-image production is that it cannot deal with rapid change in elevation. In other words, tall and large objects will hide some objects behind them due to relief displacements caused by the perspective projection and flight attitude.

A normal ortho-image is usually generated by DEM. However, DEM does not include special man-made objects such as building, road, and bridge. These objects in normal ortho-image are not usually considered in rectification process. Accidentally, a part of 
some objects that are in same elevation level with terrain can be rectified correctly. However, this effect deteriorates rather than helps the quality of ortho-image.

Another unsolved problem in normal ortho-image is so-called "double mapping" phenomenon. If a building is rectified using DSM, the building will get rectified back to its original position. However, it will also leave a copy of the building on the terrain. This is a typical "double mapping" phenomenon.

\subsection{Proposed Method for True Ortho-image Production}

One of the important issues in ortho-image production is the following question: what is the efficient method for true ortho-image production? In common terms the true ortho-image means that it tries to restore any obscured objects and to rectify all objects included in DSM correctly. A true ortho-image should be based on DSM which includes all visible objects in images. However, it is not easy task to create a perfect DSM. In general when processing true ortho-image, they are based on DSM which only include terrain, buildings, roads, and bridges.

Figure 1 shows the proposed scheme for true ortho-image production including the detailed processes.
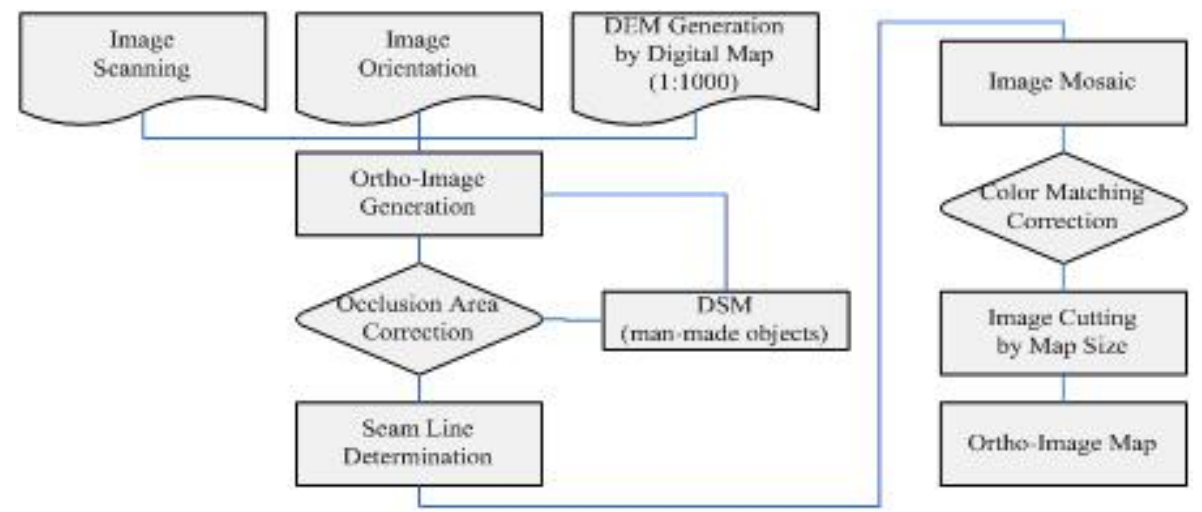

Fig. 1. The proposed scheme for true ortho-image production

Occlusion pixels detection and restoration processes are performed with the following procedure. First, the polygon for man-made objects is selected. Second, with all vertex points in object polygon, the angle for principal point on the aerial image is calculated. This angle information is used to calculate a relief displacement in later. Two points which have the maximum angle value among all vertex points are identified as a start point and an end point as shown in Figure 2. Third, relief displacements from a start vertex point to an end vertex point are calculated. Using calculated relief displacements, a new polygon for occlusion pixels can be generated on image. 


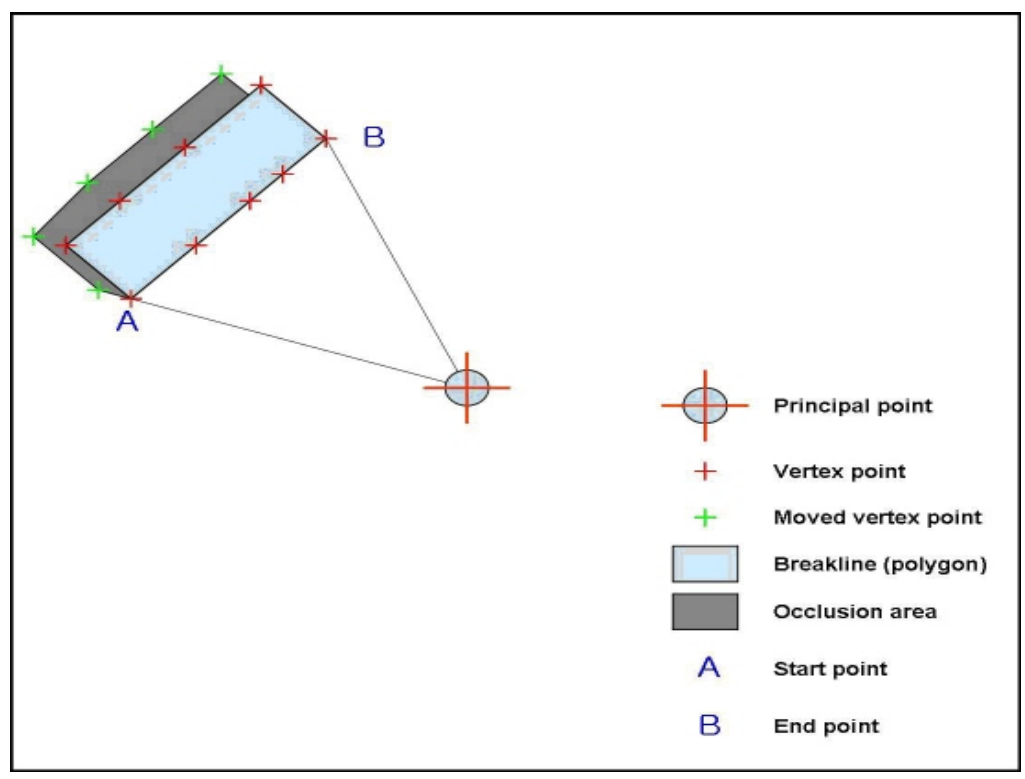

Fig. 2. Occlusion pixel detection and restoration

For occlusion pixel restoration process, radiometric equalization method is used. Correction model for color differences can be written as:

$$
D N_{\text {new }}=a \times D N_{\text {old }}+b
$$

where, $D N_{n e w}$ is a corrected pixel value, $D N_{\text {old }}$ is a original pixel value, $a$ is a gain value, and $b$ is a offset value. Finally, the feathering should be added on restored pixels.

\section{Experiments and Results}

To test the proposed true ortho-image production scheme, total 2,677 color aerial images taken in 2002 were used. Test area covers entire Seoul metropolitan city of South Korea. Since the test area covers so large range, it is impossible to solve the aerial triangulation using only one block model. Accordingly, 10 block models were used to perform the aerial triangulation as shown in Figure 3(a). Basic DEM, which has $10 \mathrm{~cm} \times 10 \mathrm{~cm}$ grid interval, for true ortho-image production was generated from 1:1,000 national digital maps. Total number of digital maps for basic DEM generation is 2,166. Figure 3(b) shows generated basic DEM in this study.

As mentioned above, true ortho-image generation should be based on DSM, not DEM. In this study, digital plotted DSM including general roads, high-level roads, bridges, and tunnels was additionally generated. Figure 4 describe full DSM for Seoul metropolitan city and detailed plot data. In case of buildings, all buildings can not be considered because the number of buildings in Seoul metropolitan city is more than 700,000 . 

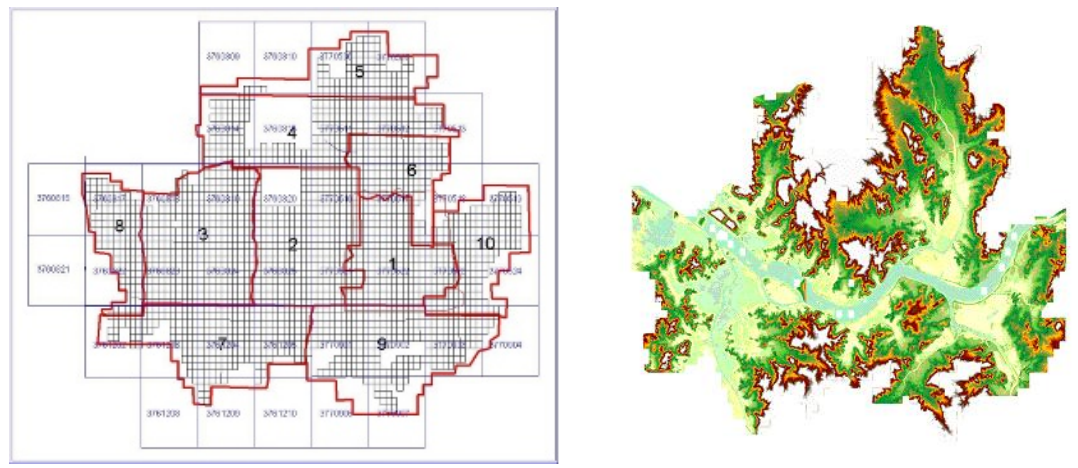

Fig. 3. 10 block models for aerial triangulation (a) basic DEM for ortho-image production (b)
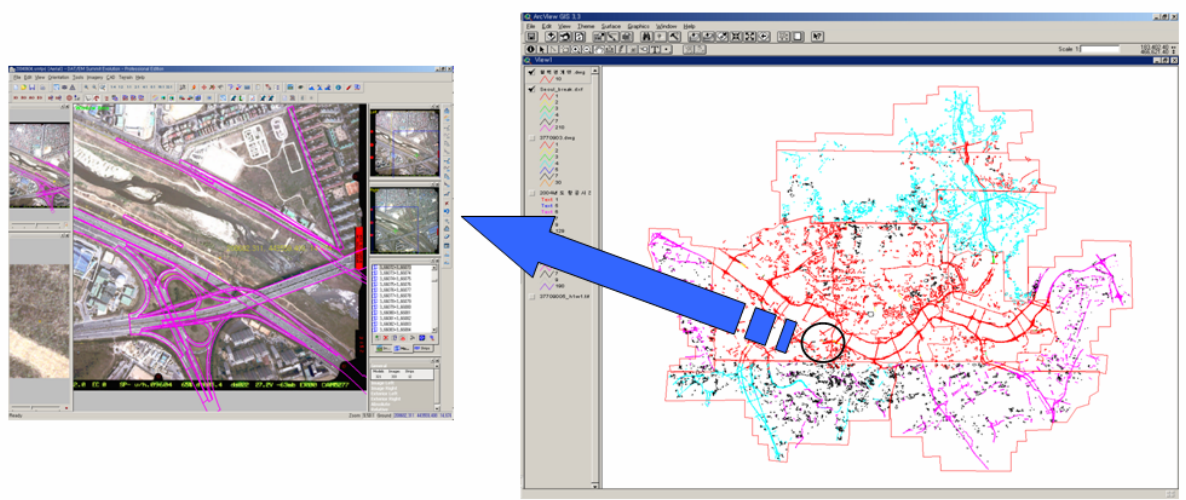

Fig. 4. Digital plotted DSM for Seoul metropolitan city

Using prepared DEM which has only terrain data and DSM which combine terrain data and digital plotted data, two ortho-rectified images were produced to evaluate the effect of true ortho-image. As shown in Figure 5, ortho-rectified image using only terrain data shows "bend down" phenomenon. However, ortho-rectified image using DSM shows corrected road image. This comparison certainly shows differences between normal ortho-image and true ortho-image.

Ortho-rectified image using DSM can solve a part of the conventional problem in ortho-image production. Nevertheless, detection and restoration for occlusion areas, which are occurred by relief displacements and fight attitude, remain to be unsolved. In order to detect occlusion pixels, all rays from the DSM to the image during orthorectification process should be checked for visibility on image and recorded. This means that ortho-rectification and occlusion pixels detection should be performed simultaneously. For restoring occlusion pixels, image of these missing pixels are needed. It can be solved by using images of the same area acquired from different locations. In aerial images for photogrammetry, this problem is naturally solved since 

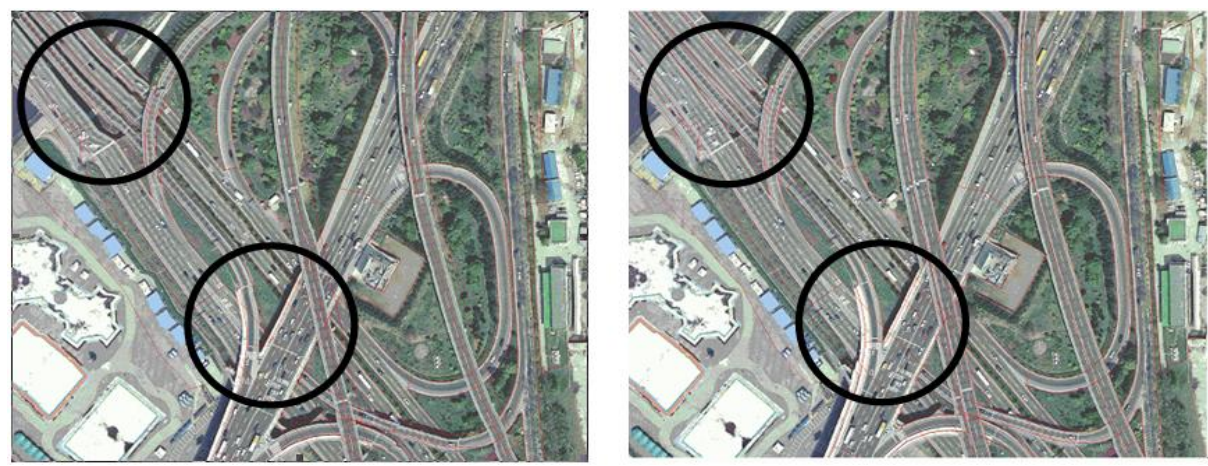

Fig. 5. Comparison between ortho-image production by DEM (a) and by DSM (b)
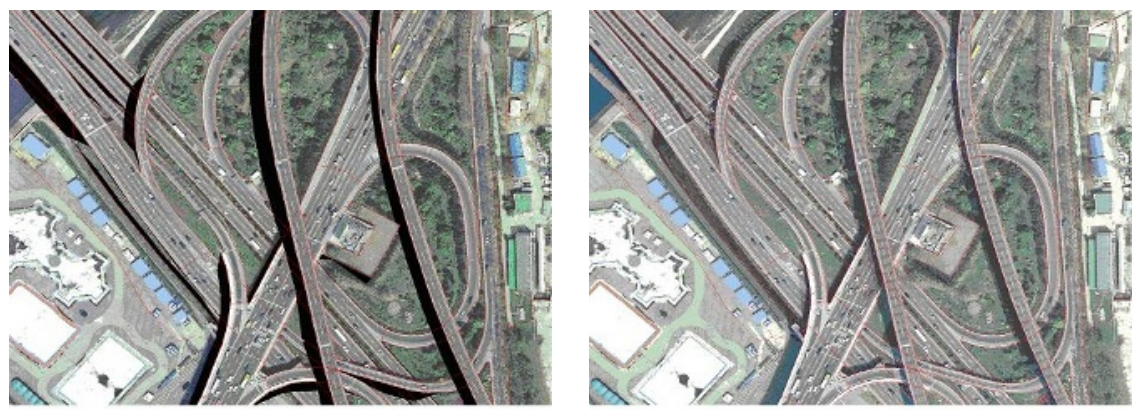

Fig. 6. Occlusion areas detection (a) and restoration (b)

aerial images are always acquired by a stereo form. Figure 6 shows occlusion pixels detection and restoration results. Black-marked pixels are occlusion areas. In this study, detection and restoration processes for occlusion pixels automatically performed through the self-developed algorithm [7].

When producing the large scale ortho-image, multiple ortho-images should be merged to form a mosaic of images. Neighboring images are usually arranged along seamlines which across along the special feature such as road in the overlapped areas. The exact route of seamlines can be determined automatically or manually. Generally, placing the seamlines along the roads often gives the best results. Figure 7(a) shows the example of ortho-image mosaic using road-based seamlines. Figure 7(b) shows color matching result in urban area after mosaic processing.

Finally, full mosaic image is cutting by the standard size such as a 1:1,000 national digital map. The reason for this is that the size of ortho-image map is the key to a successful Web GIS application. For the smooth communication between database server and client, the file size should be small if possible. In addition, image compression in this study was performed by Enhanced Compressed Wavelet (ECW) format. It is reported that ECW format theoretically allows compression of image up to 50:1 with almost no visual loss of information [8]. 

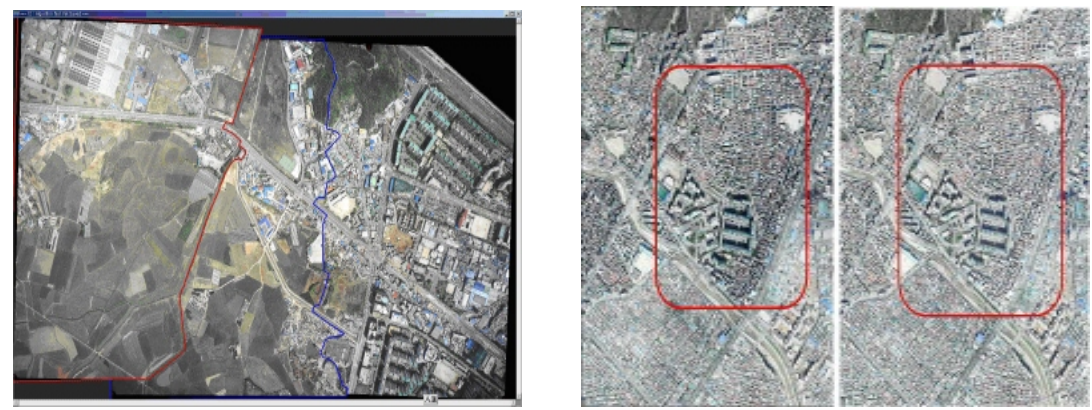

Fig. 7. Mosaic processing using road-based seamlines (a) matching result in urban area (b)

In order to evaluate the accuracy of true ortho-images generated by the proposed scheme, 7 test images are selected by considering distribution among entire orthoimages, and 20 ground check points for each image (total 140 check points) are prepared by the GPS surveying. All positional errors do not exceed $0.7 \mathrm{~m}$. This result confirms that true ortho-images in this study keep the expectation accuracy of $1 \mathrm{~m}$ in 1:1,000 national digital maps [9]. Figure 8 shows entire mosaic ortho-image for Seoul metropolitan city.

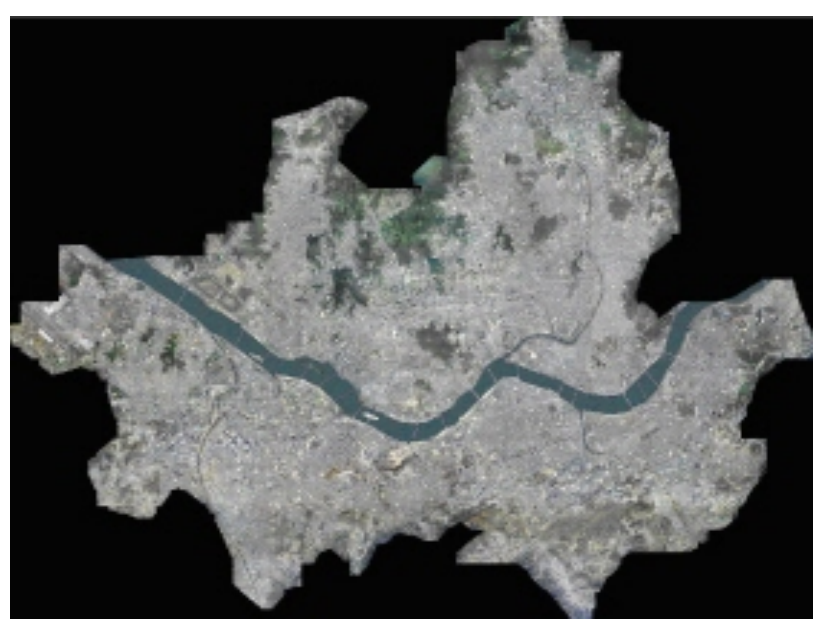

Fig. 8. Entire mosaic ortho-image for Seoul metropolitan city

\section{Conclusions}

This paper presented two main solutions regarding true ortho-image production for urban areas using aerial images: (1) Ortho-rectification technique using DSM included man-made objects (2) Occlusion area detection and restoration. We have demonstrated the proposed scheme using 2,677 aerial images containing complex artificial structures. Two following results proved that our approach was very reliable. 
First, true ortho-image generation using DSM can correct the bended linear structures such as roads and bridges in normal ortho-image. This is a serious problem that deteriorates the quality of ortho-image. Second, simultaneous processing technique for ortho-rectification and occlusion pixel detection can reduce the process time of orthoimage production. Especially, almost detected occlusion pixels can be automatically restored using the developed algorithm. For the accuracy of generated ortho-image for Seoul metropolitan city, we verify that positional errors do not exceed $0.7 \mathrm{~m}$ and it keeps the standard of 1:1,000 national digital maps.

\section{References}

1. Framework Introduction and Guide (Handbook), http://www.fgdc.gov/framework/handbook [Accessed: 4th March 2006].

2. Holm, M: An Integrated Approach for Ortho-Image Production, Photogrammetric Week '01', (2001) 249-253.

3. Novak, K.: Rectification of Digital Imagery, Photogrammetric Engineering \& Remote Sensing, 58(3), (1992) 339-344.

4. Kerschner, M.: Seamline Detection in Colour Ortho-Image Mosaicking by Use of Twin Snakes, ISPRS Journal of Photogrammetry and Remote Sensing, 56(1), (2001) 53-64.

5. Zhou, G., Schickler, W., Thorpe, A., Song, P., Chen, W., and Song, C.: True Ortho-Image Generation in Urban Areas with Very Tall Buildings, International Journal of Remote Sensing, 25, (2004) 5163-5180.

6. Kraus, K.: Photogrammetry Volume 1 Fundamentals and Standard Processes, Ferd. Dümmlers Verlag, (1993) 291-343.

7. Final Report on Color Ortho-Image Map Production Using Aerial Images, Seoul City Government, (2004).

8. ECW Product Information, http://www.es-geo.com/ecwinfo.htm [Accessed: 1st March 2006].

9. Report on the Accuracy of National Digital Basemap, National Geographic Information Institute, (1998) 41-53. 